\title{
Periodic Electric Field Enhanced Transport Through Membranes
}

\author{
T.R. Mollee ${ }^{a}$, Y.G. Anissimov ${ }^{\text {b,* }}$, M.S. Roberts ${ }^{b}$ \\ ${ }^{a}$ Department of Mathematics, University of Queensland \\ ${ }^{\mathrm{b}}$ Department of Medicine, Princess Alexandra Hospital, University of Queensland
}

\begin{abstract}
We examine the mean flux across a homogeneous membrane of a charged tracer subject to an alternating, symmetric voltage waveform. The analysis is based on the Nernst-Planck flux equation, with electric field subject to time dependence only. For low frequency electric fields the quasi steady-state flux can be approximated using the Goldman model, which has exact analytical solutions for tracer concentration and flux. No such closed form solutions can be found for arbitrary frequencies, however we find approximations for high frequency. An approximation formula for the average flux at all frequencies is also obtained from the two limiting approximations. Numerical integration of the governing equation is accomplished by use of the numerical method of lines and is performed for four different voltage waveforms. For the different voltage profiles, comparisons are made with the approximate analytical solutions which demonstrates their applicability.
\end{abstract}

Key words: periodic electric field; iontophoresis; electro-transport; membranes

\section{Introduction}

The transport of ions under the influence of an electric field is of fundamental importance to a number medical and commercial areas such as neurophysiology, iontophoresis, membrane separations and ion-exchange processes. The first theoretical treatment of electro-diffusion was by Planck [1] who assumed microscopic electro-neutrality throughout a simple electrolyte solution and modelled the transport process as a linear combination of movement due to a

* Corresponding author.

Email addresses: mollee@maths.uq.edu.au (T.R. Mollee), ya@soms.uq.edu.au (Y.G. Anissimov), mroberts@soms.uq.edu.au (M.S. Roberts). 
concentration gradient and movement due to an electromotive force acting on the ions. Plank found analytical solutions for steady state ion concentration and flux as well as the potentials that form across liquid junctions. A similar problem in membranes was studied by Goldman [2], who assumed the electric field was constant throughout the membrane.

These problems and a number of their extensions have been thoroughly reviewed by Lakshminarayanaiah $[3,4]$. The case of a time dependent electric field in the electroneutrality limit has been numerically studied by Harden \& Viovy [5] in the context of pulsed direct current (DC) iontophoresis. However mathematical treatments of electro-transport in which the electric field has time dependence appears to have had little consideration. This article provides an extension to time dependent voltages for the approximation of Goldman and extends results obtained by Keister \& Kasting [6]. We investigate the effects of symmetric, alternating voltages on mean flux of a charged species of tracer through a homogeneous, structureless membrane in the quasi steady-state. Solute transport under similar situations occurs in pulsed DC and alternating current (AC) iontophoresis.

Iontophoresis may be defined as the process of ion transport through a conducting medium due to the application of an external electric field. The external electric field is produced by electrodes of opposite polarity placed on opposite sides of the conducting medium. An ion with the same polarity as one of the electrodes is then electrostatically repelled, resulting in ion transport. The Planck and Goldman models have been applied to the study of DC iontophoresis [7].

Iontophoresis has been examined as a means of facilitating ion transport across biological and synthetic membranes [8]. In particular, transdermal iontophoresis has been explored as a means of overcoming the formidable barrier posed by the stratum corneum (SC) to penetration of charged drug species and has been found to enhance the transdermal permeation of ionic drugs several fold [9]. The constant direct currents or voltages most commonly used in these devices can be regulated, enabling the rate and duration of drug delivery to be controlled and thus offer the possibility of programmed drug delivery.

A number of side effects have been noticed with continuous DC iontophoresis including erythema, membrane polarization and electrode burns of the skin [10]. Polarisation is a result of charge stored in the skin (i.e. the SC acting like a capacitor) and results in decreased effective current, limiting the operating time of DC iontophoresis. Polarisation has been overcome to some extent by use of pulsed DC iontophoresis [11,12], however electrochemical burns at the electrodes were still present. Electrode burns are the result of $\mathrm{pH}$ changes at the electrodes due to the accumulation of hydrogen ions at the anode and hydroxide ions at the cathode [13]. Low frequency AC iontophoresis was pro- 
posed by Tapper as a method to avoid electrochemical burns at electrodes [14] and was subsequently studied by Howard et al. [13] who concluded that AC iontophoresis avoids electrochemical burns when the penetrant is Hydroxocobalamin.

High frequency AC iontophoresis has also been studied. Reinauer et al. [15] compared palmar hyperhidrosis treatment effects of DC, high frequency AC and $\mathrm{AC}$ with DC offset iontophoresis. It was found that DC and AC with DC offset iontophoresis hyperhidrosis was completely controlled. High frequency AC iontophoresis was found to have no effect. Shibaji et al. [16] studied the efficiency and voltage dependence of $\mathrm{AC}$ iontophoresis in vitro through cellophane film at high frequencies, reporting that high frequency $\mathrm{AC}$ electric field transports ions more efficiently than passive diffusion. Shibaji et al. suggest that the results of Reinauer et al. are a result of the indirect measurement method employed. Shibaji et al. theorize that the observed increase in ion transport efficiency due to the influence of an external, high frequency, alternating electric field is due to sufficient ion vibrational energy to destroy the ion-water interaction, which in turn reduces the effective stokes radius of the ion and increases the ions diffusion efficiency.

Li et al. [17] investigated effects of square wave AC iontophoresis on transport of neutral solutes through human skin. The authors assumed that during AC iontophoresis solute flux enhancement through the epidermis was due solely to the formation of new pores. Our analysis in this study demonstrates that while this assumption is most likely valid for very high frequency AC iontophoresis, for lower frequencies there is additional enhancement associated with convective motion due to the AC voltage.

The analysis of tracer concentration and flux presented here is based on conservation of mass and the Nernst-Plank electrodiffusion equation with a time dependent electric field. The electric field is assumed to be proportional to the applied voltage. The voltage waveforms considered are square, triangular, trapezoidal and sinusoidal. Comparisons between average flux results are made at equivalent single-phase mean voltages for the different voltage profiles. Section 2 provides a discussion of the model. In section 3 results of numerical integration for the membrane ion concentration and average flux through the membrane for the different voltage profiles are discussed and the accuracy of approximations obtained from these results are examined. Of particular interest is the relative insensitivity of the approximation to the voltage waveform. Explicit representations of the voltage waveforms and the approach for obtaining the approximations are given in the appendices. We note that the analysis presented in this article applies to homogeneous membranes and so may not be directly applicable to the heterogeneous stratum corneum [18]. 


\section{The Model}

We consider a homogeneous membrane separating a donor electrolyte solution containing a tracer species of ions and a receiver solution consisting of the same electrolyte as in the donor, but which is tracer free. It is assumed that these solutions are well stirred, semi-infinite media held at constant concentrations of tracer, counter and co-ions. An electrode, connected to an AC power supply, is placed in each solution (see Figure 1) generating an alternating electric field across the membrane. We further assume that intrinsic membrane properties are not altered when transmembrane potentials exist.

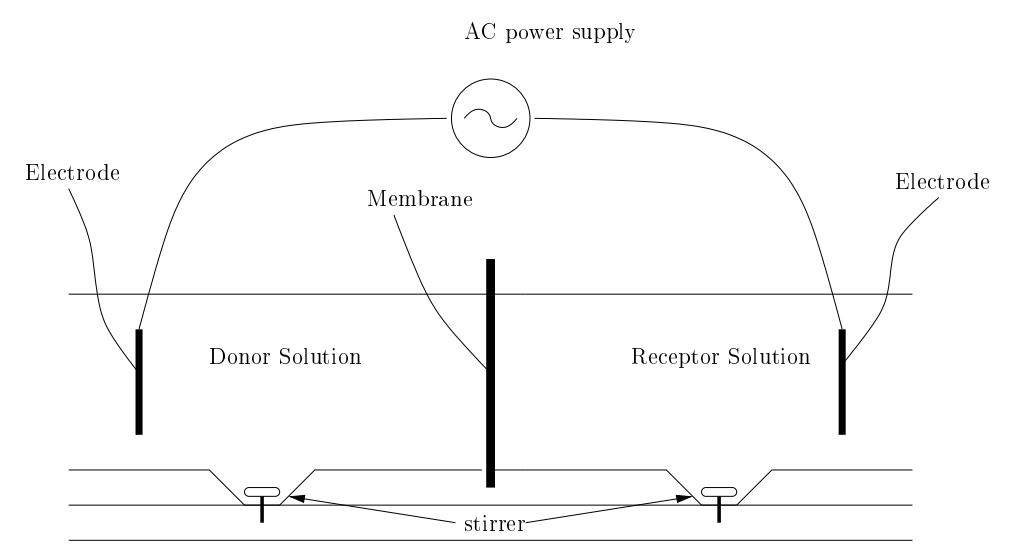

Fig. 1. Schematic diagram of AC electro-transport through a membrane. The solution to the left contains tracer and electrolyte, and the solution to the right contains electrolyte and is held at zero tracer concentration. The stirrers keeps the solutions well stirred.

Consider the one dimensional transport of tracer ions through a membrane oriented perpendicular to the $x$-direction, of thickness $h$ and initially tracer free, subject to an external, time dependent, periodic voltage $V(t)$. Transport of tracer is investigated using conservation of mass and the Nernst-Planck flux equation. The respective equations are $[9,19]$

$$
\begin{gathered}
\frac{\partial C}{\partial t}(x, t)=-\frac{\partial J}{\partial x}(x, t) \\
J(x, t)=-D \frac{\partial C}{\partial x}(x, t)+(z \mu E+(1-\sigma) \nu) C(x, t)
\end{gathered}
$$

where $C(x, t)$ is the tracer concentration in the membrane, $J$ is the tracer flux in the membrane, $E$ is the electric field inside the membrane, $D$ is the tracer diffusion coefficient in the membrane, $\mu$ is the electrophoretic mobility of tracer ions in the membrane, $z$ is the tracer valency, $\sigma$ is the reflection coefficient of the membrane to tracer ions, and $\nu$ is the velocity of convective water flow due to electro-osmosis.

The electric field across the membrane is assumed to depend only on time and 
is related to the potential across the membrane, $V(t)$ by

$$
E(t)=\frac{V(t)}{h} .
$$

We write the voltage as $V(t)=V_{0} \Lambda(t)$, where $\Lambda(t)$ is a periodically varying function of unit amplitude and $V_{0}$ is the voltage waveforms amplitude.

Furthermore, we assume that the magnetic field generated by the time dependent electric field, a consequence of the Maxwell equations of electromagnetism, has no appreciable effect on tracer transport.

On combining (1), (2) and (3) the tracer concentration satisfies the partial differential equation

$$
\frac{\partial C}{\partial t}(x, t)=D \frac{\partial^{2} C}{\partial x^{2}}(x, t)-v(t) \frac{\partial C}{\partial x}(x, t), \quad 0 \leq x \leq h, \quad \text { and } \quad t>0,
$$

with

$$
v(t)=v_{0} \Lambda(t), \quad \text { and } \quad v_{0}=v_{e l}+v_{o s}=\frac{D z e V_{0}}{k T h}+(1-\sigma) V_{0} k_{1}
$$

where $v_{e l}$ is the velocity amplitude due to electro-transport and $v_{o s}$ is the velocity amplitude due to electro-osmotic transport. Further $e$ is the charge of an electron, $k$ is Boltzmann's constant and $T$ is the local temperature in the Kelvin scale. In (5) Einstein's relation $D=\mu k T$, for an idealized system was used [19] and $\nu$ was assumed to be proportional to $V(t)$, $\nu(t)=k_{1} V(t)$, with $k_{1}$ positive or negative.

In (5) the velocity due to electro-transport $\left(v_{e l}\right)$ is usually much greater than the velocity arising from electro-osmosis $\left(v_{o s}\right)$. In the stratum corneum the ratio $v_{o s} / v_{e l}$ could be approximated by the ratio of permeability coefficients $(P C)$ of a neutral solute to that of a positively charged one under identical voltage or current conditions. Using human skin iontophoresis data from Yoshida \& Roberts [20] for neutral sucrose and positively charged lignocaine (which have similar molar volumes) one gets $v_{o s} / v_{e l} \approx P C_{\text {suc }} / P C_{l i g}=0.05$. Due to the relatively small contribution from electro-osmosis, in what follows the effects of electro-osmosis are not taken into account. However for neutral solutes $v_{e l}=0$, and $v_{0}$ is solely determined by electro-osmosis, in which case electro-osmosis can not be neglected.

Equation (4) is subject to boundary conditions on tracer concentration just inside the membrane at $x=0$ and $x=h$. To simplify the analysis, we assume the membrane-solution ion partitioning coefficient for the tracer to be unity. The boundary conditions we examine are

$$
C(0, t)=C_{0} \quad \text { and } \quad C(h, t)=0 \quad \text { for } \quad t>0,
$$




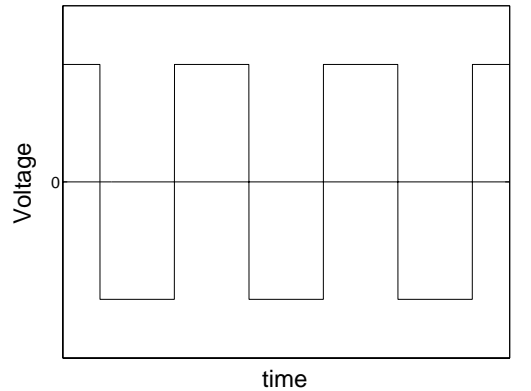

(a)

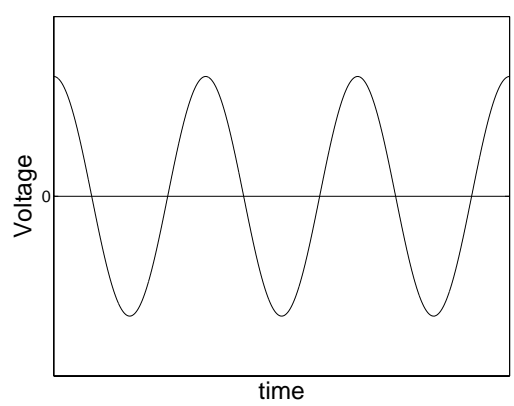

(c)

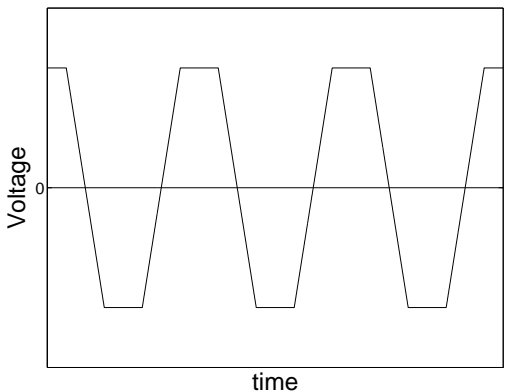

(b)

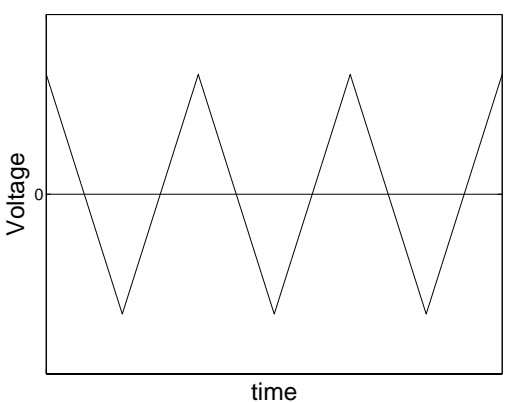

(d)

Fig. 2. Voltage waveforms used in this study driving electro-transport across a membrane. Square (a), trapezoidal (b), sine (c) and triangular (d) waveforms.

where $C_{0}$ is the constant tracer concentration of the donor. Keister \& Kasting [6] integrated (4) for a constant electric field $(\Lambda(t)=1)$, finding the time dependent solution.

The voltage waveforms considered are a modified square wave, triangular wave, trapezoidal wave and sine wave (see Appendix A and Figure 2). The use of a modified square wave in place of a square wave will be discussed in Section 3.

If $f$ is the frequency of the waveform the mean voltage, $\bar{V}$, over the positive phase of the voltage is

$$
\bar{V}=2 f \int_{0}^{1 /(2 f)} V(t) d t .
$$

In future this will be referred to simply as the mean voltage. Mean voltages for the modified square, triangular, trapezoidal and sine wave profiles of Appendix $\mathrm{A}$ are $99 V_{0} / 100, V_{0} / 2,3 V_{0} / 4$ and $2 V_{0} / \pi$ respectively. This magnitude gives a measure of how strongly tracer ions are influenced by the electric field during one phase of a cycle. Comparison of results between the different waveforms is then meaningful for equivalent mean voltages.

To simplify later calculations we introduce the two parameters $t_{d}=h^{2} / D$ and $\alpha=h v_{0} \bar{V} /\left(V_{0} D\right)$. The first has dimension of time and is the characteristic timescale when only diffusion drives transport. The second is dimensionless, 
being the ratio of $t_{d}$ to the mean time taken for a tracer ion with velocity $v(t)$ to travel the length of the membrane during the electric field's positive phase. It is then a measure of the relative dominance of electro-transport to diffusion during DC iontophoresis. It can also be written $\alpha=\bar{V} e z /(k T)$, using (5). For a tracer of unit valency in a solution at a temperature of $30^{\circ} C, \alpha=38.29 \bar{V}$ (where $\bar{V}$ is in volts).

A quasi-steady state is reached when the transient component of the tracer concentration is negligible, leaving only a periodic function of time, and the system is independent of its initial condition.

The average tracer flux at position $x$ in the quasi-steady state can be written

$$
\langle J\rangle=\lim _{t_{0} \rightarrow \infty} f \int_{t_{0}}^{t_{0}+f^{-1}} J(x, t) d t .
$$

Averaging (1) over a period in the quasi steady state and using the periodicity of $C(x, t)$ we have

$$
\frac{\partial}{\partial x}\langle J\rangle=0
$$

Hence the average flux in quasi-steady state is independent of position.

The amount of tracer passing through the cross-sectional area $A$ at the membrane receptor boundary from time zero to $t$ is given by

$$
Q(t)=A \int_{0}^{t} J(h, \tau) d \tau
$$

The average flux can then be written

$$
\langle J\rangle=\lim _{t_{0} \rightarrow \infty} \frac{f}{A}\left(Q\left(t_{0}+f^{-1}\right)-Q\left(t_{0}\right)\right)
$$

Characteristic lengths for ion transport due to the electric field and diffusion (passive transport) for a frequency $f$ are respectively

$$
\delta_{1}=\int_{0}^{1 /(2 f)} v(\tau) d \tau \quad \text { and } \quad \delta_{2}=\sqrt{\frac{D}{f}} .
$$

$\delta_{1}$ is a measure of distance travelled by a tracer ion subject to the action of the electric field during the positive phase of one voltage cycle, and $\delta_{2}$ is a measure of the distance travelled by a tracer ion due to diffusion over one voltage period.

Define the characteristic frequency $f^{*}$ as the frequency for which $\delta_{1}=h$. In terms of the effective voltage for the waveforms considered here $f^{*}$ may be 
written

$$
f^{*}=\frac{v_{0} \bar{V}}{2 h V_{0}}=\frac{\alpha}{2 t_{d}} .
$$

The quantity $1 /\left(2 f^{*}\right)$ can be interpreted as the mean time that a tracer ion takes to just cross the entire membrane due to electro-transport alone.

Assuming that ions traverse the stratum corneum through appendages [21] and that the diameter of the ions is sufficiently small to permit transport through these pores, taking $h=15 \mu \mathrm{m}$ and the diffusion coefficient $D=10^{-6} \mathrm{~cm}^{2} \mathrm{~s}^{-1}$, for a system subject to a voltage waveform with $\bar{V}=1$ volt results in $f^{*}=8.5 \mathrm{~Hz}$. This value is rather large and may be the result of assuming that an ion traverses the membrane through a straight pore. If a tortuous path is taken then $h$ may increase by one to two orders of magnitude which would result in $f^{*}$ being reduced by two to four orders of magnitude.

In terms of this characteristic frequency $\delta_{1}$ and $\delta_{2}$ may be written

$$
\delta_{1}=h \frac{f^{*}}{f} \quad \text { and } \quad \delta_{2}=h \sqrt{\frac{2 f^{*}}{\alpha f}} .
$$

If $f \gg f^{*}$, both $\delta_{1}$ and $\delta_{2}$ are much less than the membrane thickness so that the mean distance travelled over half a period by a tracer ion is much less than the membrane thickness.

Approximations for $\langle J\rangle$ will be discussed for small $\left(f \ll f^{*}\right)$ and large $(f \gg$ $\left.f^{*}\right)$ frequencies. For large frequencies we present approximations for cases in which electro-transport or diffusion are the dominant boundary transport processes.

Electro-transport dominates near the boundaries when $\delta_{2} \ll \delta_{1} \ll h / 2$. This restricts the available frequencies for transport of this nature to the range $f^{*} \ll f \ll \alpha f^{*} / 2$. For a monovalent species of tracer ion these inequalities correspond to $10 f^{*} \lesssim f \lesssim \alpha f^{*} / 40$, with $\alpha \gtrsim 800$. For temperatures around $30^{\circ} \mathrm{C}$ this requires $\bar{V} \gtrsim 20$ volts.

Diffusion dominating near the boundary is equivalent to $\delta_{1} \ll \delta_{2} \ll h / 2$. This requires the frequency to satisfy $f \gg f^{*} \max \{\alpha / 2,8 / \alpha\}$.

\section{Results and discussion}

The numerical integration of (4) was performed by reducing the problem to non-dimensional form and then following the numerical method of lines [22], 


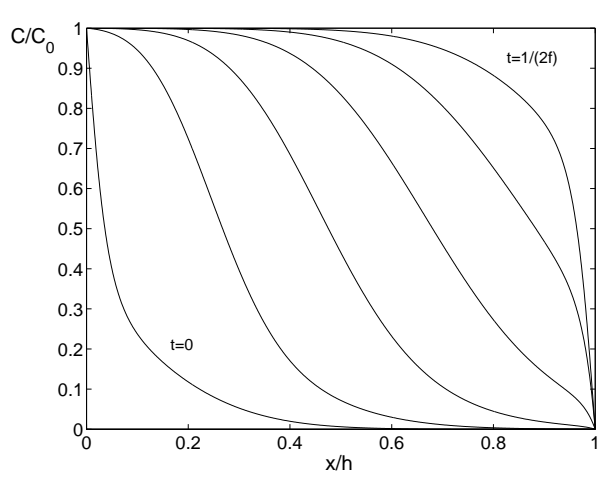

(a)

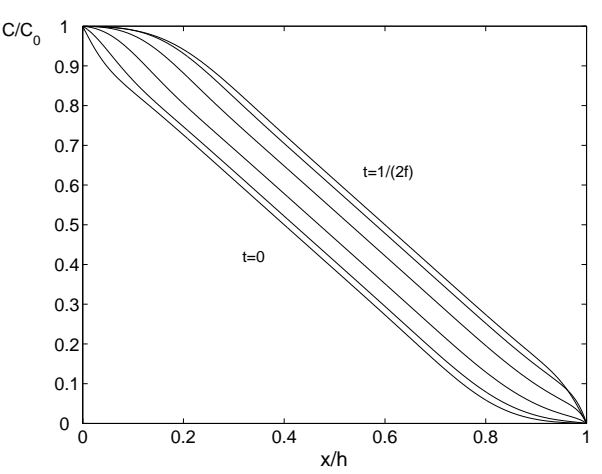

(b)

Fig. 3. Quasi steady-state concentration profiles during the positive voltage phase. Figure (a) is for square wave voltage with $\bar{V}=1$ volt and frequency $f=f^{*}$. Figure (b) is for a sine wave voltage with $\bar{V}=1$ volt and frequency $f=5 f^{*}$. In each figure the curve to the furthest left corresponds to the beginning of the positive voltage phase, with subsequent curves at increments of $1 /(10 f)$.

which is a common method for solving parabolic partial differential equations. The method involves breaking (4) into a system of first order, ordinary differential equations in $t$ by discretising the spatial variable. Fourth order finite difference were used to approximate the spatial derivatives. The resulting system was then integrated using the numerical package CVODE (from Lawrence Livermore National Laboratory, available from http://www.llnl.gov/), the integrations being performed on a Linux cluster. Average flux was found using the trapezoidal rule at $x=h / 2$ and $x=h$.

Evaluating $\langle J\rangle$ at both $x=h / 2$ and $x=h$ allowed a check of the value obtained. When a discontinuous square wave was used there were large discrepancies in the $\langle J\rangle$ values. This was overcome by using a modified square wave (Appendix A).

At effective voltages of $0.5,1.0$, and 2.0 volts, results were obtained with uniform spatial discretisation involving 1000 nodes. For each effective voltage the average flux for each profile was found at 65 values of $f / f^{*}$, distributed for frequencies $0.05 f^{*} \leq f \leq 150 f^{*}$.

Figure 3a shows 5 plots of concentration in quasi steady-state starting at the beginning of the positive phase of the square wave voltage with $\bar{V}=1$, and finishing at the end of the positive phase. A similar set of curves were observed during the negative phase of the voltage. For frequencies $0.05 f^{*} \leq f \leq f^{*}$ all concentration profiles for all voltage waveforms show the same behavior of periodic movement between the curve to the farthest left and the one to the farthest right.

For a modified square wave voltage an approximation for $\langle J\rangle$, obtained in 
Appendix B, is

$$
\langle J\rangle=\frac{J_{\text {pass }} \alpha}{2}\left[1-\frac{f}{f^{*}}\left(1-\frac{3}{\alpha}\right)\right], \quad \text { where } \quad J_{\text {pass }}=\frac{C_{0} D}{h} .
$$

The accuracy of the linear approximation for a modified square wave with $\bar{V}=1$ for frequencies $0<f<0.7 f^{*}$ can be seen in Figure 4a. A comparison of (13) with the results of numerical integration gives an error in flux enhancement of less than $5 \%$, where flux enhancement is defined as $\langle J\rangle-J_{\text {pass }}$. It is surprising that (13) is accurate over so large a frequency range, considering the approximation was obtained by assuming $f \ll f^{*}$.

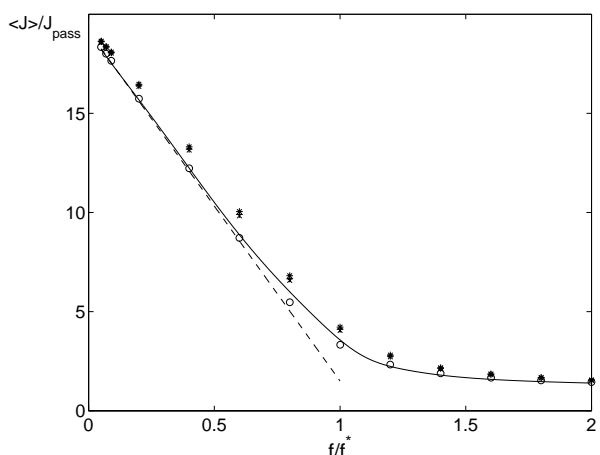

(a)

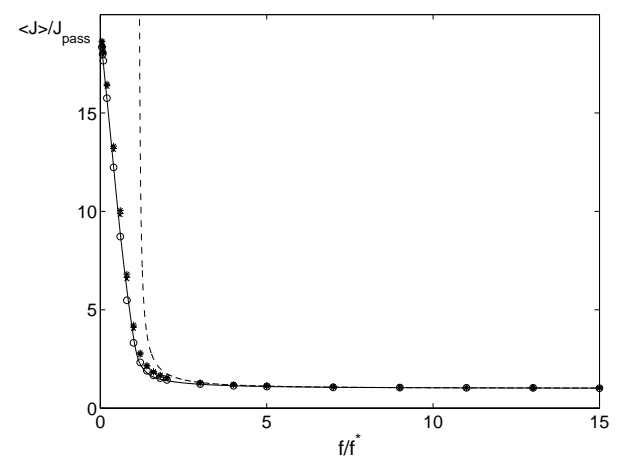

(b)

Fig. 4. Average flux results for the four voltage waveforms with $\bar{V}=1$ volt. The $\mathrm{o}, \times,+$ and $*$ correspond to square, trapezoidal, sine and triangular numerical calculations respectively and the continuous lines correspond to Equation (16) with $\gamma=0.18$ and $\rho=0$. Figure (a) includes the linear approximation (13) valid for small frequencies (--). Figure (b) Equation (15) (--).

Similar results hold for the modified square wave voltage for the remaining mean voltages. For $\bar{V}=0.5$ and 2.0 volts comparison of (13) with the numerical integration results for $0.05 f^{*} \leq f \leq 0.7 f^{*}$ gives an enhancement error less than $5 \%$.

In Figure 4a the numerical integration values for average flux for trapezoidal, sine and triangular waveforms appear to be linear with their gradient differing from that of a modified square wave voltage. We considered the following generalization of (13)

$$
\langle J\rangle=\frac{J_{\text {pass }} \alpha}{2}\left[1-\frac{f}{f^{*}}\left(1-\frac{\rho}{\alpha}\right)\right],
$$

where $\rho$ alters the gradient of the line and is determined by comparing the results of numerical integration with (14) for each waveform. 


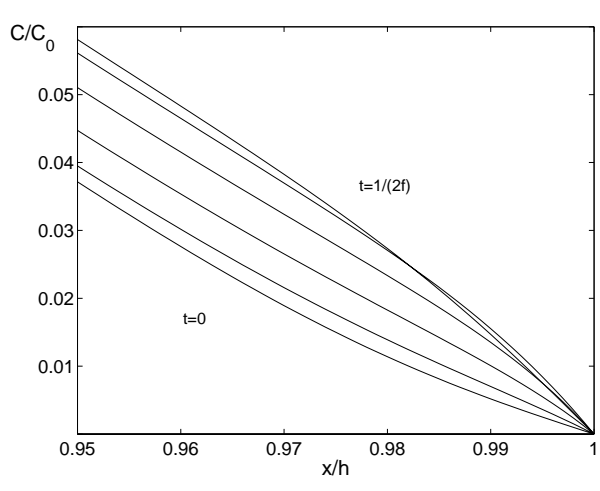

(a)

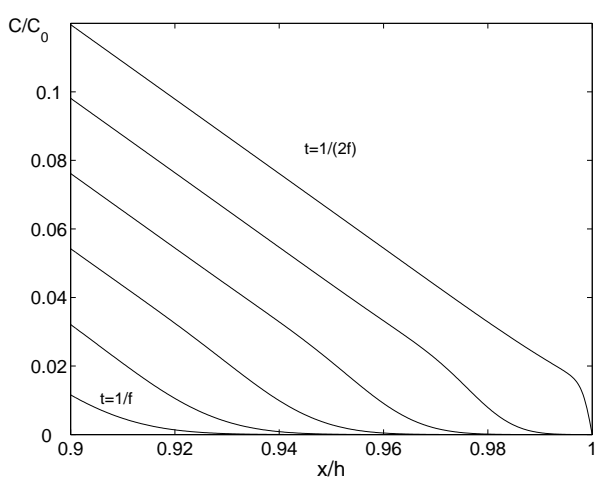

(b)

Fig. 5. Quasi steady-state concentration curves at the membrane receptor boundary. Each curve corresponds to time increments of $1 /(10 f)$. Figure (a) is for a sine wave voltage with $\bar{V}=1$ volt and frequency $f=50 f^{*}$ during the positive phase of the voltage. Figure (b) is for a modified square wave voltage with $\bar{V}=25$ volts and frequency $f=10 f^{*}$ during the negative phase of the voltage.

For trapezoidal, sine and triangular voltage waveforms (14) with $\rho=7.0$ was found to be accurate to error less than $5 \%$ for $f$ between 0 and $0.5 f^{*}$.

We now consider electro-transport when frequencies $f \gg f^{*}$ and transport such that diffusion is the dominant boundary process. Figure $3 \mathrm{~b}$ shows concentration profiles for a sine wave voltage with frequency $f=5 f^{*}$. The curves are confined to a narrow strip and appear linear away from the boundaries with slopes slightly greater than 1 in magnitude. This behavior is observed in all concentration profiles for the different voltage waveforms considered.

Figure 5a displays concentration profiles close to the membrane-receptor boundary when the voltage waveform is a sine wave with large frequency $\left(f=50 f^{*}\right)$ and $\bar{V}=1$.

An approximation for $\langle J\rangle$, obtained in Appendix $\mathrm{C}$, is

$$
\langle J\rangle=\frac{J_{\text {pass }}}{1-\gamma \sqrt{\frac{\alpha f^{* 3}}{f^{3}}}},
$$

where $\gamma$ is a constant which is to be determined by comparing the numerical integration results with (15) for the different waveforms.

Approximation (15) approaches 1 asymptotically with increasing $f$. This is expected physically since at very high frequencies electro-transport, represented by the second term on the right hand side of (4), is negligible, leaving the external concentration gradient driving tracer ion transport, hence $\langle J\rangle=J_{\text {pass }}$. Taking $\gamma=0.2$ for a modified square wave voltage results in flux enhancement errors less than $10 \%$ for the frequency and voltage regimes $f>5 f^{*}$ at $\bar{V}=0.5, f>7 f^{*}$ at $\bar{V}=1.0$, and $f>14 f^{*}$ at $\bar{V}=2.0$. This increase in 
the lower bound of the frequency range with increasing mean voltage (and therefore $\alpha$ ) at which approximation (15) is valid is expected as the derivation of (15) assumed $f \gg f^{*} \alpha / 2$. For the remaining voltage waveforms $\gamma=0.3$ in (15) gives the flux enhancement error less than $10 \%$ for the mean voltages considered and frequency $f>50 f^{*}$. The absolute error in approximation (15) however is small for low to moderate frequencies for all voltage waveforms. This can be seen in Figure 4b which displays numerical integration results for all four voltage waveforms with $\bar{V}=1$ and approximation (15) for a square wave voltage with moderate to low frequencies.

Based on approximations (14) and (15) we derived the following approximation valid for each waveform considered here (at low mean voltages) and for all frequencies:

$$
\langle J\rangle=\frac{J_{\text {pass }}\left(1+\gamma \sqrt{\alpha / \varphi^{3}}\right)}{1+1.4 \sqrt{\alpha} / \varphi^{14}}+\frac{\alpha J_{\text {pass }}}{2\left(1+0.25 \varphi^{10}\right)\left(1+\varphi(1-\rho / \alpha)+0.2 \alpha^{0.7} \varphi^{3}\right)},
$$

where $\varphi=f / f^{*}$. Equation (16) approaches (14) and (15) for small and large frequencies respectively and with $\gamma=0.18$ and $\rho=0$ is used to approximate the average flux for the square wave voltage. The remaining waveforms (sinusoidal, trapezoidal and triangular) are approximated with $\gamma=0.27$ and $\rho=$ 14. The relative errors resulting from using (16), with the appropriate $\gamma$ and $\rho$ values, for the voltages considered here, $\bar{V}=0.5,1$ and 2 volts, are less than $15 \%$.

When electro-transport is the dominant boundary transport process the numerical integration was performed with 2000 equally spaced spatial nodes. For the voltages considered there was a large difference between $\langle J\rangle$ at $x=h / 2$ and $\langle J\rangle$ at $x=h$. For a modified box wave this large difference was eliminated by taking 4000 equally spaced nodes, however the time required to run these cases was excessive. There was no significant difference between the calculated $\langle J\rangle$ at $x=h / 2$ for the different cases.

Numerical integration shows concentration profiles to be linear over the majority of the membrane. However there is rapid change near the membrane boundaries, indicating the presence of boundary layers. The region near $x=h$ is shown in Figure 5b. We note the slope of the curves are negligible compared with that for times close to $1 /(2 f)$.

An approximation for $\langle J\rangle$ when electro-transport dominates transport near the membrane boundaries is (see Appendix $\mathrm{C}$ for derivation)

$$
\langle J\rangle=\frac{J_{\text {pass }}}{1-f^{*} / f+\sqrt{2 \pi f^{*} /\left(\alpha v^{2} f\right)}} .
$$


For a modified square wave taking $v=1.4$ gives flux enhancement errors less than $5 \%$ in the appropriate frequency ranges for both $\bar{V}=25$ and 40 volts. For trapezoidal, sine and triangular waveforms, the respective values $v=1.9$, 1.6 and 2.0 all give enhancement errors less than $5 \%$ over the appropriate frequency ranges for both $\bar{V}=25$ and 40 volts.

We have analyzed the experimental data of Shibaji et al. [16] for AC iontophoresis of $\mathrm{NaCl}$ across a cellophane membrane. The relevant physical parameters here are $h=36 \mu \mathrm{m}, D=3.510^{5} \mathrm{~cm}^{2} \mathrm{~s}^{-1}, T=37^{\circ} \mathrm{C}$ and $f=10^{3} \mathrm{~Hz}$ and the voltage waveform is a sine wave with effective voltages 10,15 and 20 volts. These conditions lead to $f^{*}$ values of 505, 758 and $1011 \mathrm{~Hz}$ for 10, 15 and 20 volts respectively. As these values are not covered by the approximations developed in this paper the data was analyzed by numerical integration. The results are $\langle J\rangle=1.81 J_{\text {pass }},\langle J\rangle=3.28 J_{\text {pass }}$ and $\langle J\rangle=17.35 J_{\text {pass }}$ for the 10, 15 and 20 volt cases respectively and are consistent with an enhancement of flux by AC iontophoresis as reported by Shibaji et al., but the theoretical predictions are somewhat less than the experimental observations. A key outcome therefore, recognized by this analysis for the first time, is that AC electro-transport contributes to the AC iontophoretic flux.

The asymptotic analysis presented here to obtain approximations (14), (15) and (17) allows clarification of the physical basis for AC iontophoretic flux enhancement. For low frequencies, $f \ll f^{*}$, enhancement is largely due to ion transport during the positive voltage cycles, and as the frequency goes to zero the flux approaches half the iontophoretic flux of the equivalent DC voltage iontophoresis. For high frequencies, $f \gg f^{*}$, quasi steady-state concentrationdistance profiles in the membrane away from boundary regions are linear, similar to passive diffusion. In this case the apparent thickness $\left(h_{\text {app }}\right)$ of the membrane is reduced, $h_{\text {app }} \approx h\left(1-\gamma \sqrt{a\left(f^{*} / f\right)^{3}}\right)$ for (15) and $h_{a p p} \approx h\left(1-f^{*} / f\right)$ for (17). This reduction of apparent membrane thickness is due to the presence of boundary layers around $x=0$ and $x=h$, which arise from combined periodic convective motion and diffusion of ions.

\section{Conclusion}

The problem of finding average flux across a membrane of a charged tracer subject to an alternating, symmetric voltage waveform was considered in this work. Approximate solutions to average flux were derived and found to agree well with the results of numerical integration. We found that for low frequency 
periodic electric fields average flux is significantly enhanced compared to the passive diffusion flux, while for high frequencies the average flux rapidly approaches that for passive diffusion. The results of this analysis could be applied to $\mathrm{AC}$ iontophoresis through human skin providing that new pore induction in stratum corneum is also taken into account [17].

\section{Acknowledgements}

The authors acknowledge the support of the National Health and Medical Research Council of Australia and the Queensland and New South Wales Lions Medical Research Foundation. 


\section{List of Symbols}

A Cross-sectional area of membrane

C Tracer concentration in membrane

$C_{0} \quad$ Tracer concentration in donor

D Tracer diffusion coefficient in membrane

$e \quad$ Charge of electron

E $\quad$ Electric field acting on membrane

$f \quad$ Frequency of voltage

$f^{*} \quad$ Characteristic frequency

$h \quad$ Thickness of membrane

$J \quad$ Tracer flux

$J_{\text {pass }}$ Passive steady-state tracer flux

$\langle J\rangle \quad$ The average flux in quasi stead-state

$k \quad$ Boltzmann's constant

$Q(t)$ Amount of tracer passing through cross-section of membrane

$t \quad$ Time

$t_{d} \quad$ Characteristic diffusion time scale

$T \quad$ Temperature

$v(t) \quad$ Tracer velocity

$v_{0} \quad$ Peak tracer velocity

$V(t) \quad$ Voltage

$V_{0} \quad$ Voltage amplitude

$\bar{V} \quad$ Mean voltage

$x \quad$ Spatial coordinate

$z \quad$ Valency of tracer 


$\begin{array}{ll}\alpha & \text { Dimensionless constant } \\ \gamma & \text { Numerical parameter (dimensionless) } \\ \delta_{1} & \text { Average distance travelled by tracer due to } E \\ \delta_{2} & \text { Characteristic distance travelled by tracer due to diffusion } \\ \Lambda(t) & \text { Periodic function with unit amplitude } \\ \mu & \text { Electrophoretic mobility of tracer in membrane } \\ \nu & \text { Velocity of convective water flow } \\ \rho & \text { Numerical parameter (dimensionless) } \\ \sigma & \text { Reflection coefficient of membrane to tracer ions } \\ v & \text { Numerical parameter (dimensionless) }\end{array}$

A

Voltage Waveforms

The modified square voltage waveform $\Lambda_{s q r}$, with unit amplitude and frequency $f$ is defined for $0 \leq t \leq 1 / f$ by

$$
\Lambda_{s q r}(t)=\left\{\begin{array}{lll}
200 f t & \text { for } & 0<t<\frac{1}{200 f} \\
1 & \text { for } & \frac{1}{200 f}<t<\frac{99}{200 f} \\
100-200 f t & \text { for } & \frac{99}{200 f}<t<\frac{101}{200 f} \\
-1 & \text { for } & \frac{101}{200 f}<t<\frac{199}{200 f} \\
200 f t-200 & \text { for } & \frac{199}{200 f}<t<\frac{1}{f}
\end{array}\right.
$$

The trapezoidal voltage waveform $\Lambda_{t r p}$, with unit amplitude and frequency $f$ is defined for $0 \leq t \leq 1 / f$ by

$$
\Lambda_{t r p}(t)=\left\{\begin{array}{lll}
8 f t & \text { for } & 0<t<\frac{1}{8 f} \\
1 & \text { for } & \frac{1}{8 f}<t<\frac{3}{8 f} \\
4-8 f t & \text { for } & \frac{3}{8 f}<t<\frac{5}{8 f} \\
-1 & \text { for } & \frac{5}{8 f}<t<\frac{7}{8 f} \\
8 f t-8 & \text { for } & \frac{7}{8 f}<t<\frac{1}{f}
\end{array}\right.
$$


The triangular voltage waveform $\Lambda_{t r i}$, with unit amplitude and frequency $f$ is defined for $0 \leq t \leq 1 / f$ by

$$
\Lambda_{t r i}(t)=\left\{\begin{array}{lll}
4 f t & \text { for } & 0<t<\frac{1}{4 f} \\
2-4 f t & \text { for } & \frac{1}{4 f}<t<\frac{3}{4 f} \\
4 f t-4 & \text { for } & \frac{3}{4 f}<t<\frac{1}{f}
\end{array}\right.
$$

The sine voltage waveform $\Lambda_{\text {sin }}$, with unit amplitude and frequency $f$ is defined by

$$
\Lambda_{\text {sin }}(t)=\sin (2 \pi f t)
$$

B

Consider a square wave voltage with frequency $f \ll f^{*}$. Figure 3 a suggests, and numerics confirm, that during the positive phase of the voltage the tracer concentration profile comes very close to the steady state solution $C^{+}(x)$ of the problem

$$
\frac{\partial C}{\partial t}(x, t)=D \frac{\partial^{2} C}{\partial x^{2}}(x, t)-v_{0} \frac{\partial C}{\partial x}, \quad \text { with } \quad C(0, t)=C_{0} \quad \text { and } \quad C(h, t)=0,
$$

which is

$$
C^{+}(x)=C_{0} e^{v_{0} x / 2 D} \frac{\sinh \left(v_{0}(h-x) / 2 D\right)}{\sinh \left(h v_{0} / 2 D\right)} .
$$

Numerics also confirm that during the negative phase of the voltage the system evolves to come very close to the steady state solution $C^{-}(x)$ of the problem

$$
\frac{\partial C}{\partial t}(x, t)=D \frac{\partial^{2} C}{\partial x^{2}}(x, t)+v_{0} \frac{\partial C}{\partial x}, \quad \text { with } \quad C(0, t)=C_{0} \quad \text { and } \quad C(h, t)=0
$$

which is

$$
C^{-}(x)=C_{0} e^{-v_{0} x / 2 D} \frac{\sinh \left(v_{0}(h-x) / 2 D\right)}{\sinh \left(h v_{0} / 2 D\right)} .
$$

In order to approximate the amount of solute $Q$ transported out of the membrane and into the receptor over one period, we divide the problem into finding the amount out during the time when $V(t)>0$, labelled $Q^{+}(1 / 2 f)$, and the amount out during the time when $V(t)<0$, labelled $Q^{-}(1 / 2 f) \cdot Q^{+}(1 / 2 f)$ is found from (B.1) with the initial condition $C^{-}(x)$. Similarly $Q^{-}(1 / 2 f)$ is found from (B.3) and the initial condition $C^{+}(x)$. The only difference between these problems is the sign preceding $v_{0}$. Thus once $Q^{+}(1 / 2 f)$ is known, $Q^{-}(1 / 2 f)$ follows with the change $v_{0}$ to $-v_{0}$. 
Equation (B.1) with initial condition $C^{-}(x)$ has an exact solution in the Laplace domain. Denote the Laplace transform in time of the function $C(x, t)$ by $\hat{C}(x, s)$, where $s$ is the transform variable. The solution for $\hat{C}$ is

$$
\begin{aligned}
\hat{C}(x, s)= & A_{1}(s) e^{-(1-x / h)\left(\alpha+\sqrt{\alpha^{2}+4 t_{d} s}\right) / 2}+A_{2}(s) e^{-(1-x / h)\left(\alpha-\sqrt{\alpha^{2}+4 t_{d} s}\right) / 2} \\
& +B_{1}(s) e^{-\alpha x / h}+B_{2}(s)
\end{aligned}
$$

where

$$
\begin{aligned}
& A_{1}(s)=\frac{C_{0} \alpha^{2}\left(e^{\alpha}-e^{-\left(\alpha-\sqrt{\alpha^{2}+4 t_{d} s}\right) / 2}\right)}{2 s\left(s t_{d}-2 \alpha^{2}\right) \sinh (\alpha / 2) \sinh \left(\sqrt{\alpha^{2}+4 t_{d} s} / 2\right)} \\
& A_{2}(s)=-\frac{C_{0} \alpha^{2}\left(e^{\alpha}-e^{-\left(\alpha+\sqrt{\alpha^{2}+4 t_{d} s}\right) / 2}\right)}{2 s\left(s t_{d}-2 \alpha^{2}\right) \sinh (\alpha / 2) \sinh \left(\sqrt{\alpha^{2}+4 t_{d} s} / 2\right)} \\
& B_{1}(s)=\frac{C_{0} t_{d}}{\left(s t_{d}-2 \alpha^{2}\right)\left(1-e^{-\alpha}\right)} \quad \text { and } \quad B_{2}(s)=-\frac{C_{0} e^{-\alpha}}{s\left(1-e^{-\alpha}\right)}
\end{aligned}
$$

The Laplace transform of $(2)$ at $x=h$ is

$$
\hat{J}(h, s)=-D \frac{\partial \hat{C}}{\partial x}(h, s) .
$$

¿From (B.5) we have then

$$
\begin{aligned}
\hat{J}(h, s)= & \frac{C_{0} v_{0} e^{\alpha / 2}}{2 s\left(t_{d} s-2 \alpha^{2}\right) \sinh (\alpha / 2)}\left[\alpha \sqrt{\alpha^{2}+4 t_{d} s} \operatorname{coth}\left(\sqrt{\alpha^{2}+4 t_{d} s} / 2\right)\right. \\
& \left.+\left(\alpha^{2}+t_{d} s\right)-\frac{\alpha e^{\alpha} \sqrt{\alpha^{2}+4 t_{d} s}}{\sinh \left(\sqrt{\alpha^{2}+4 t_{d} s} / 2\right)}\right] .
\end{aligned}
$$

This is a singled valued analytic function of $s$ with a removable singularity at $s=2 \alpha^{2} / t_{d}$ and simple poles at $s=0$ and at $s=-\alpha^{2} /\left(4 t_{d}\right)-k^{2} \pi^{2} / t_{d}$ for all positive integers $k$. From this function $J_{s s}^{+}$the steady state flux for positive voltages at $x=h$ can be found.

The simple poles occurring for $\Re(s)<0$ correspond to transitive terms in $J(h, t)$ which tend to zero as $t$ becomes large. This leaves only the singularity 
at $s=0$ to consider for large times $t$, which will contribute a constant term to the expression for the boundary flux. Evaluating the residue at $s=0$ gives

$$
J_{s s}^{+}=\lim _{s \rightarrow 0}[s \hat{J}(h, s)]=\frac{C_{0} v_{0}}{1-e^{-\alpha}} \approx C_{0} v_{0} \quad \text { when } \quad e^{\alpha} \gg 1
$$

The Laplace transform of (9) is

$$
\hat{Q}(s)=\frac{A}{s} \hat{J}(h, s) .
$$

This is a single valued analytic function of $s$ with a removable singularity at $s=2 \alpha^{2} / t_{d}$ and simple poles at $s=-\alpha^{2} /\left(4 t_{d}\right)-k^{2} \pi^{2} / t_{d}$ for all positive integers $k$. However there is now a second order pole at $s=0$ which, upon inversion, will contribute a term linear in $t$ plus a constant term to the expression for $Q^{+}(t)$. The simple poles along $\Re(s)<0$ again correspond, in the inversion, to transient terms which decay rapidly with increasing $t$.

We take as an approximation for $Q^{+}(t)$ the contribution from the pole at $s=0$ in the inversion of $\hat{Q}^{+}(s)$. Hence

$$
\begin{aligned}
Q^{+}(t) & \approx \lim _{s \rightarrow 0} \frac{d}{d s}\left[s^{2} \hat{Q}(s) e^{s t}\right] \\
& =\frac{C_{0} A v_{0}}{1-e^{-\alpha}}\left[t-\frac{t_{d}}{2 \alpha^{2}}\left(3 \alpha \operatorname{coth}(\alpha / 2)-\alpha-e^{-\alpha}-5\right)\right] .
\end{aligned}
$$

The first transient term in $Q^{+}(t)$ is

$$
\frac{256 C_{0} v_{0} t_{d} \alpha \pi^{2} e^{\alpha} \operatorname{coth}(\alpha / 2)}{\left(\alpha^{2}+4 \pi^{2}\right)^{2}\left(9 \alpha^{2}+4 \pi^{2}\right)} e^{-\left(\alpha^{2} / 4+\pi^{2}\right) t / t_{d}} .
$$

For $1 /\left(2 f^{*}\right)<t<5 /\left(2 f^{*}\right)$ the ratio of this term to the constant term in $Q^{+}(t)$ is of order $10^{-6}$ for a system of monovalent ions at $30^{\circ} \mathrm{C}$. The subsequent terms in the transient component of $Q^{+}(t)$ decay more rapidly than this, justifying the linear approximation we have taken for large $t$.

Replacing $v_{0}$ with $-v_{0}$ in $Q^{+}(t)$ gives

$$
Q^{-}(t) \approx \frac{C_{0} A v_{0}}{1-e^{-\alpha}}\left[t e^{-\alpha}-\frac{t_{d}}{2 \alpha^{2}}\left(3 \alpha e^{-\alpha} \operatorname{coth}(\alpha / 2)+\alpha e^{-\alpha}-5 e^{-\alpha}-1\right)\right] .
$$

Then $Q$ is the sum of $Q^{+}(t)$ and $Q^{-}(t)$ taken over half a period each, 


$$
\begin{aligned}
Q & \approx C_{0} A v_{0}\left[\frac{\operatorname{coth}(\alpha / 2)}{2 f}-\frac{3 t_{d}}{2 \alpha} \operatorname{coth}^{2}(\alpha / 2)+\frac{t_{d}}{2 \alpha}+\frac{3 t_{d}}{\alpha^{2}} \operatorname{coth}(\alpha / 2)\right] \\
& =\frac{A C_{0} v_{0}}{2 f}\left[1-\frac{f}{f^{*}}\left(1-\frac{3}{\alpha}\right)\right]+O\left(e^{-\alpha}\right) .
\end{aligned}
$$

Hence for a square wave voltage, when $f \ll f^{*}$ the time averaged boundary flux of tracer can be approximated by

$$
\langle J\rangle=\frac{f Q}{A} \approx \frac{C_{0} v_{0}}{2}\left[1-\frac{f}{f^{*}}\left(1-\frac{3}{\alpha}\right)\right] \text { where } e^{\alpha} \gg 1
$$

\section{C}

This section determines an approximation for $\langle J\rangle$ for the symmetric, alternating voltage profiles of Appendix A for frequencies satisfying $f \gg f^{*}$.

Consider the system to have evolved to quasi steady-state and that diffusion is the dominant process near the boundary. From Figure $3 \mathrm{~b}$ the concentration away from the boundary layers appears to be linear and to oscillate horizontally with time. Since we are in quasi steady-state we can consider the time variable to start at $t=0$ when the voltage waveforms are at the mid-point of the positive phase. The concentration profile corresponding to $t=0$ will have linear section passing through the point $\left(h / 2, C_{0} / 2\right)$ of slope slightly greater in magnitude to that for the equivalent problem with diffusion acting alone.

If we were to extend the boundaries to infinity so that they cannot affect the concentration, a quasi-steady solution to (4) is

$$
C(x, t)=A-B(x-\delta(t)), \quad \text { where } \quad \delta(t)=\int_{0}^{t} v(\tau) d \tau
$$

and $A$ and $B$ are constants. It appears then that the concentration at $x=h / 2$ in Figure $3 \mathrm{~b}$ does not feel the effects of the boundaries. So we take (C.1) as the solution to (4) in a neighborhood of $x=h / 2$.

With this solution, and (2), an expression for the mean flux is

$$
\langle J\rangle=f \int_{0}^{1 / f} j(h / 2, t) d t=f B \int_{0}^{1 / f}(D+v(t) \delta(t)) d t .
$$

The contribution from the second term in the integrand is zero as $v(t)=$ $d \delta(t) / d t$ over appropriate intervals of integration for the different wave forms. Hence $\langle J\rangle=B D$ where $B$ is unknown. 


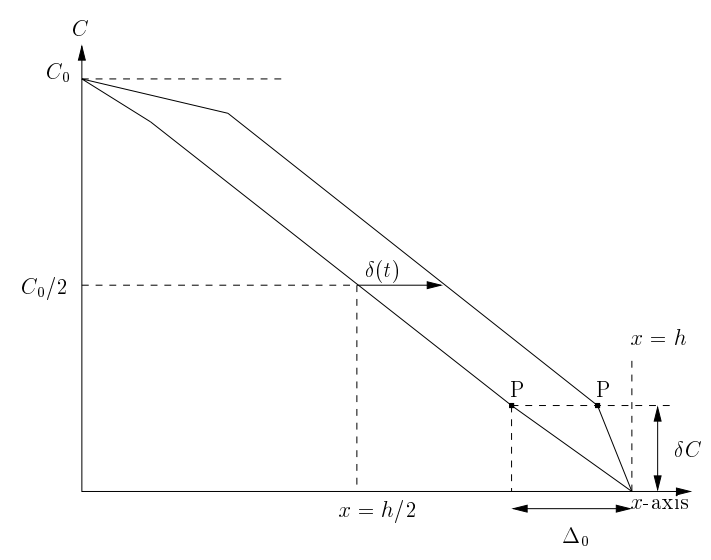

(a)

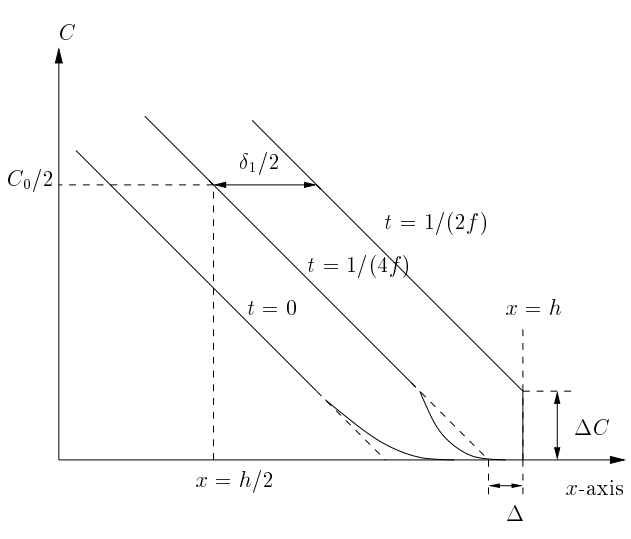

(b)

Fig. C.1. Idealized concentration curves used to obtain average flux approximations (15) and (17) for (a) diffusion and (b) electro-transport being the dominant boundary transport process.

To find an expression for $B$ we start by approximating the concentration profiles, such as in Figures $3 \mathrm{~b}$ and $5 \mathrm{a}$, by piecewise linear segments as shown in Figure C.1a.

¿From Figure 5a, $B$ is easily seen to be $B=\left(C_{0}-2 \delta C\right) /\left(h-2 \Delta_{0}\right)$. Hence an expression for the average flux is

$$
\langle J\rangle=\frac{D\left(C_{0}-2 \delta C\right)}{h-2 \Delta_{0}} .
$$

where $\Delta_{0}$ and $\delta C$ are unknown. To determine $\Delta_{0}$ and $\langle J\rangle$ another expression for $\langle J\rangle$ is found in the boundary layer near $x=h$. From these two expressions an approximation for $\langle J\rangle$ can be found.

Consider viewing the transport process at a point moving with the particles (point $P$ in Figure C.1a such that the concentration remains constant $(\delta C)$, and yet such that changes behind $P$ in the linear section and changes near the boundary can be observed. At $t=0$ label this distance from the boundary $\Delta_{0}$. We expect $\Delta_{0}$ to be proportional to $\delta_{2}$, i.e. $\Delta_{0}=\beta \delta_{2}$.

The slope of the linear approximation in the boundary layer is time dependent and is of magnitude

$$
\frac{\delta C}{\Delta_{0}-\delta(t)}
$$

The average flux is

$$
\langle J\rangle=D f \delta C I \quad \text { where } \quad I=\int_{0}^{1 / f} \frac{d t}{\Delta_{0}-\delta(t)} .
$$


Eliminating $\delta C$ in (C.3) and (C.5) yields the expression

$$
\langle J\rangle=\frac{C_{0} D}{h-2 \Delta_{0}+2 / f I} .
$$

For an initial sine wave the integral $I$ (after a change of variables) is given in [23], and assuming $\delta_{1}<2 \Delta_{0}$ results in

$$
I=\frac{2}{f \sqrt{4 \Delta_{0}^{2}-\delta_{1}^{2}}} .
$$

Hence

$$
\frac{1}{I}=f \Delta_{0}\left(1-\frac{\delta_{1}^{2}}{8 \Delta_{0}^{2}}+O\left(\frac{\delta_{1}^{4}}{\Delta_{1}^{4}}\right)\right)
$$

and using this in (C.6)

$$
\langle J\rangle \approx \frac{C_{0} D}{h-\delta_{1}^{2} / 4 \Delta_{0}}
$$

This can be written

$$
\langle J\rangle \approx \frac{J_{\text {pass }}}{1-\frac{1}{4 \beta} \sqrt{\alpha f^{* 3} /\left(2 f^{3}\right)}} .
$$

The approximation is completed by finding the value for $\beta$ so that (C.10) agrees with the numerical integration results. The fraction $1 /(4 \sqrt{2} \beta)$ we write as $\gamma$. The same process yields, for the remaining voltage waveforms, an equation of the same form with different $\gamma$ values.

Next, consider electro-transport to be the dominant boundary process. Numerical integration shows the concentration is linear away from the boundary layers. The slope in the boundary layers is steep for times around $t=1 /(2 f)$ due to the magnitude of the mean voltages considered. Figure C.1b shows thew situation in general, though not to scale. $\Delta$ is the distance between the end of the linear section of the concentration curve at $t=1 /(4 f)$ and the boundary $x=h$, while $\Delta C$ is the height of the concentration curve at $t=1 /(2 f)$ between the end of the linear section and $C=0$.

The central sections of the concentration curves is again taken to be of the form (C.1). From Figure C.1b, the slope of the concentration away from the boundary layers is $B \approx C_{0} /(h-2 \Delta)$, and hence

$$
\langle J\rangle \approx \frac{C_{0} D}{h-2 \Delta}
$$

where $\Delta$ is to be determined. To determine $\langle J\rangle$ and $\Delta$ we once again find an expression for $\langle J\rangle$ in the boundary layer and use the two expressions to solve for $\langle J\rangle$. 
To approximate the net flux through $x=h$ consider sitting at a point a distance $\delta(t)$ from the boundary $x=h$ such that we are near the end of the concentration's linear section and can observe the events near the boundary. At times for which $V(t)<0$ tracer ions are being pulled towards the donor strongly, leaving the region near the boundary at very low concentration. During this time tracer ions diffuse into this boundary region. These particles do not feel the effects of the boundary due to the strength of $V(t)$. When the sign of $V(t)$ changes all particles that diffused into the boundary region are forced through $x=h$. Hence finding the net flux through $x=h$ of tracer is proportional to the amount out, $Q(t)$, for the problem

$$
\frac{\partial C}{\partial t}(x, t)=D \frac{\partial^{2} C}{\partial x^{2}}(x, t)
$$

for $0 \leq x<\infty, t>0, C(x, 0)=0$ and $C(0, t)=\Delta C$, where $\Delta C$ is the constant concentration at the end of the linear section we are viewing the process from.

The solution to (C.12) is given by Crank [24] and is

$$
C(x, t)=\Delta C \operatorname{erf}_{c}\left(\frac{x}{\sqrt{4 D t}}\right)
$$

where $\operatorname{erf}_{c}(\cdot)$ is the complementary error function. The amount out per unit area is then

$$
\frac{Q(t)}{A}=\Delta C \sqrt{\frac{4 D t}{\pi}}
$$

From Figure C.1b it can be seen that

$$
\Delta C \approx C_{0} \frac{\left(\delta_{1}-2 \Delta\right)}{2(h-2 \Delta)}
$$

Hence the average flux through $x=h$ is approximated by

$$
\langle J\rangle \approx \frac{v f Q(1 / f)}{f}=\frac{C_{0}\left(\delta_{1}-2 \Delta\right) v}{2(h-2 \Delta)} \sqrt{\frac{4 D f}{\pi}},
$$

where $v$ is a dimensionless parameter to be determined by comparing (C.13) with the results of numerical integration for each waveform.

Solving simultaneously (C.11) and (C.13) results in

$$
\Delta \approx \frac{\delta_{1}}{2}-\sqrt{\frac{\pi}{4 v^{2}}} \delta_{2}
$$

and 


$$
\langle J\rangle \approx \frac{C_{0} D}{h-\delta_{1}+\frac{\sqrt{\pi}}{v} \delta_{2}}=\frac{J_{\text {pass }}}{1-f^{*} / f+\sqrt{2 \pi f^{*} /\left(\alpha v^{2} f\right)}}
$$

\section{References}

[1] M. Planck, Über die erregung von elektrizität und wärme in elektrolyten, Ann. Phys. Chem. 39 (1890) 161-186.

[2] D. Goldman, Potential impedance and rectification in membranes, Journal of general physiology 27 (1943) 37-60.

[3] N. Lakshminarayanaiah, Transport phenomena in membranes, Academic Press, Inc, 1969, Ch. 3.

[4] N. Lakshminarayanaiah, Equations of membrane biophysics, Academic press, 1984 .

[5] J. Harden, J. Viovy, Numerical studies of pulsed iontophoresis through model membranes, Journal of Controlled Release 38 (1996) 129-139.

[6] J. Keister, G. B. Kasting, Ionic mass transport through a homogeneous membrane in the presence of a uniform electric field, Journal of Membrane Science 29 (1986) 155-167.

[7] G. B. Kasting, Theoretical models for iontophoretic delvery, Advanced drug delivery reviews 9 (1992) 177-199.

[8] P. Singh, H. Maibach, Iontophoresis in drug delivery: basic principles and application, Critical reviews in therapeutic drug carrier systems 11 (1994) 161213.

[9] A. K. Banga, Electrically assisted transdermal and topical drug delivery, Taylor and Francis Group, 1998.

[10] P. W. Ledger, Skin biological issues in electrically enhanced transdermal delivery, Advanced drug delivery reviews 9 (1992) 289-307.

[11] S. Odia, E. Vocks, J. Rakoski, J. Ring, Successful treatment of dyshidrotic hand eczma using tap water iontophoresis with pulsed direct current, Acta dermatovenereologica 76 (1996) 472-474.

[12] M. Pikal, S. Shah, Study of the mechanisms of flux enhancement through hairless mouse skin by pulsed dc iontophoresis, Pharmaceutical research 8 (1991) 365-369.

[13] J. Howard, T. Drake, D. Kellogg, Effects of alternating current iontophoresis on drug delivery, Archives of physical medicine and rehabilitation 76 (1995) $463-466$. 
[14] R. Tapper, Iontophoretic treatment system, U.S. Patent, patent Number $5,224,927$ (1993).

[15] S. Reinauer, A. Neusser, G. Schauf, E. Hölzle, Iontophoresis with alternating current and direct current offset (ac/dc iontophoresis): a new approach for the treatment of hyperhidosis, Brittish journal of dermatology 129 (1993) 166-169.

[16] T. Shibaji, Y. Yasuhara, N. Oda, M. Umino, A mechanism of the high frequency ac iontophoresis, Journal of controlled release 73 (2001) 37-47.

[17] S. Li, A. Ghanem, K. Peck, W. Higuchi, Pore induction in human epidermal membrane during low to moderate voltage iontophoresis: A study using ac iontophoresis, Jornal of Pharmaceutical Sciences 88 (4) (1999) 419-427.

[18] Y. Anissimov, M. Roberts, Diffusion modeling of percutaneous absorption kinetics: 3. variable diffusion and partition coefficients, consequences for stratum corneum depth profiles and desorption kinetics, Journal of pharaceutical sciences 93 (2004) 470-487.

[19] G. Giebisch, D. Tosteson, H. Ussing (Eds.), Membrane transport in biology, Springer-Verlag, 1978, Ch. 2, pp. 5-113.

[20] N. Yoshida, M. Roberts, Solute molecular size and transdermal iontophoresis across excised human skin, Journal of controlled release 25 (1993) 177-195.

[21] C. Cullander, What are the pathways of iontophoretic current flow through mammalian skin?, Advanced Drug Delivery Reviews 9 (1992) 119-135.

[22] W. Schiesser, The numerical method of lines: integration of partial differential equations, Academic Press, New York, 1991.

[23] G. F. Carrier, M. Krook, C. E. Pearson, Functions of a Complex Variable, McGraw-Hill,Inc, 1966, Ch. 3, pp. 86-87.

[24] J. Crank, The Mathematics of Diffusion, Oxford, 1956. 